\title{
Wirtschaftlich, nachhaltig, digitalisiert
}

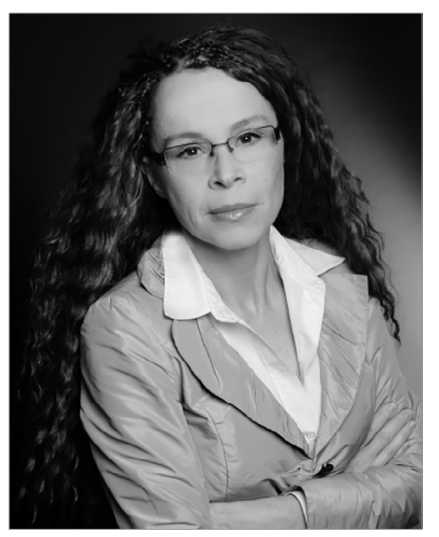

VON GABRIELE HELLER

Gabriele Heller gründete im Jahre 2005 die Firma Heller-Consulting $\mathrm{GmbH}$ als Beratungsunternehmen in der Sozialwirtschaft mit dem Schwerpunkt Beschaffungsoptimierung. Davor war sie als Finanzreferentin bei einem freien Träger der Evang.--Luth. Kirche in Bayern tätig.

www.heller-consulting.net

\author{
Ein Produkt zum niedrigen Einkaufspreis zu bestellen \\ ist einfach. Das gesamte Beschaffungswesen \\ jedoch so umzustellen, dass es viele Bedingungen \\ wie niedrige Personalkosten, Regionalität, faire \\ Produktqualität, pünktliche Lieferung, geringe \\ Lagerkosten, zuverlässige Verfügbarkeit und \\ Nachhaltigkeit erfüllt, bedeutet für sozialwirtschaftliche \\ Unternehmen einen besonderen Kraftakt.
}

Sozialunternehmen entwickeln heute verstärkt ein Bewusstsein dafür, wie entscheidend wertschöpfend der Einkauf für eine positive Bilanz eines Unternehmens ist. Leider fehlen oft die Personalressourcen und die Zeit, sich auch noch um die optimale Beschaffung von Verbrauchsartikeln zu kümmern. Und selbst wenn diese Ressourcen vorhanden sind, fehlt manchmal das notwendige spezifische »Einkaufs-Know-how«. Und selbst wenn dieses im Unternehmen vorhanden ist, koordiniert werden sollen und auch das Rechnungsmanagement effizienter organisiert werden soll, dann wird der Einkauf komplexer. Im Zeitalter der Digitalisierung bedeuten komplexere Prozesse mehr Daten und neue Formen des Datenmanagements.

Am Markt vorhandene Programme zur Einkaufsoptimierung gibt es als Insellösungen, andere Programme arbeiten mit einem kompletten Portfolio entlang eines Source-to-Pay-Prozesses.

\section{"Besonders Komplexträger können im Einkauf schnell ihre Kosteneffizienz verbessern"}

verändern sich die Einkaufsprozesse vor der Kulisse der Digitalisierung.

Aber das Kostenbewusstsein ist zunehmend vorhanden, welches Einkaufsverantwortliche veranlasst, die Lieferantenauswahl zu optimieren und vertraute Beschaffungsstrukturen $\mathrm{zu}$ hinterfragen. Wenn eine größere Zahl von potenziellen Lieferanten gewünscht ist, um mehr Preise vergleichen zu können, wenn die Bestellprozesse besser
Sozialwirtschaftliche Komplexträger mit ihrem Mix an verschiedenen Einrichtungen und Diensten können im Einkauf relativ schnell ihre Kosteneffizienz verbessern und Kosten reduzieren. Eingesetzt werden im modernen Beschaffungsmanagement bestimmte Module, die Kunden helfen, nicht nur jeden einzelnen Prozessabschnitt zu managen, sondern die Prozessschritte auch beliebig und reibungslos zu kombinieren, damit 
Kosten einzusparen und gleichzeitig Abläufe deutlich zu vereinfachen. Für die Mitarbeiter im Einkauf bringt »die Digitalisierung « Arbeitserleichterung und verhilft der ganzen Abteilung zu einem messbaren, verbesserten Ergebnisbeitrag für das Unternehmen.
Lieferanten-, Bestell- und Rechnungsmanagement zählt, aus Ausbildung und Studium bekannte Modelle, über deren Bedeutung man nicht mehr diskutieren muss. Moderne Management-Systeme, die einzelne Segmente bis hin zur Gesamtheit einer Source-to-Pay-Kette op-

\section{"Größter Stolperstein beim digitalisierten Einkauf ist die Akzeptanz durch die Mitarbeitenden"}

\section{Einkaufsstrategie}

Das sozialwirtschaftliche Unternehmen beschafft nur dann optimal, wenn die Warengruppen strukturiert sind. Häufig gibt es bei Trägern noch eine hohe Fragmentierung von Artikeln, Daten und Artikelstämmen. Da holt jeder Einrichtungsstandort seine eigenen Angebote ein, hat seine eigenen Lieferanten und rechnet mit diesen wie gewohnt ab. So werden unzählige Belege in Papierform produziert, wodurch hohe Prozesskosten verursacht werden und vor allem auch im Unternehmen niemand so recht weiß, wo Ineffizienzen versteckt sind. Ein Beschaffungsbenchmark innerhalb des Trägers lässt sich unter diesen Bedingungen unmöglich ermitteln.

Gute digitale Beschaffungsprogramme schaffen im ersten Schritt erst einmal Transparenz und einen Überblick. Alle Rechnungen, Lieferscheine und Bestellungen werden in das System eingelesen und in die Logik optimierter Einkaufsprozesse transformiert.

Die Erfahrungen bei der Einführung von digitalisierten Beschaffungsprozessen zeigen allerdings, dass nicht die Fachsoftware und transportierten Prozessdefinitionen das zentrale Problem darstellen. Die am Markt vorhandenen Programme haben einen Reifegrad erreicht, der auf der betriebswirtschaftlichen und datentechnischen Seite relativ unkomplizierte Auswahlentscheidungen ermöglicht.

Die Stolpersteine bei der Umstellung auf den digitalisierten Einkauf liegen, wie sollte es auch anders sein, in der Akzeptanz bei den Mitarbeitenden.

Für junge Nachwuchskräfte im Einkaufs- und Logistikmanagement sind Supply-Chain-Modelle, zu denen das timieren, sind normales Handwerkszeug - mit enormen Einsparpotenzialen bei den Prozesskosten.

Doch die Umstellung auf ein effizienteres Beschaffungswesen, so unsere Erfahrung, gelingt besser, wenn das Projekt exemplarisch an einem Teilbereich startet, dort zeigen kann, dass strukturierte Beschaffung machbar und betriebswirtschaftlich erfolgreich funktioniert. Durch einen Piloten, zum Beispiel »Lebensmitteleinkauf «, gelingt es auch besser, skeptische, in alten Routinen verhaftete Mitarbeitende für das neue System zu gewinnen.

\section{Beispiel Lebensmitteleinkauf}

Der Lebensmitteleinkauf ist auch ein emotionaler Bereich, der viel mit persönlichen Kontakten, mit lokaler und regionaler Nähe zu tun hat. Doch wie kann man »Bauchgefühle « durch Argumente ersetzen?

In einem solchen Entscheidungsprozess gibt es nichts Belastbareres als Fakten; und die liefert das Bestell-System. Es bietet objektive Ergebnisse, nach denen entschieden werden kann. Ergebnisse, die im Grunde auch die einzigen relevanten Kriterien bilden müssen. Der Einkäufer bestellt weiterhin von seinem Standort aus, bekommt aber die Top-Konditionen eines Großeinkäufers. Bei der Warengruppe der Lebensmittel konnten alle Beteiligten schnell die Vorteile eines neuen Einkaufssystem erkennen, so dass die Geschäftsleitung davon überzeugt war, auch weitere Warengruppen in strukturierte Abläufe zu überführen. Nach den Lebensmitteln kamen beispielsweise Reinigungsmittel, Büromaterial, Werkzeuge oder Arbeitskleidung hinzu.

Transparenz in allen Bereichen ist ein entscheidender Faktor ist, da mehr bekannte Zahlen auch mehr Transparenz bedeuten und damit eine wesentliche Entscheidungsgrundlage bilden.

Die digitale Transformation des Einkaufs sollte also weiterhin an den vorhandenen Strukturen ansetzen und dezentral aufgebaut werden, so dass niemanden »etwas weggenommen wird «. Dem Einkauf Transparenz zu verschaffen, macht im Unternehmen eigene Ressourcen frei und beschleunigt Prozesse im Unternehmen. Und bei Einkaufdimensionen eines großen Trägers erreicht man bei den $\mathrm{He}$ belwarengruppen Bereiche, bei denen beim Einkauf Menge zählt.

\section{Einkauf in der Sozialwirtschaft}

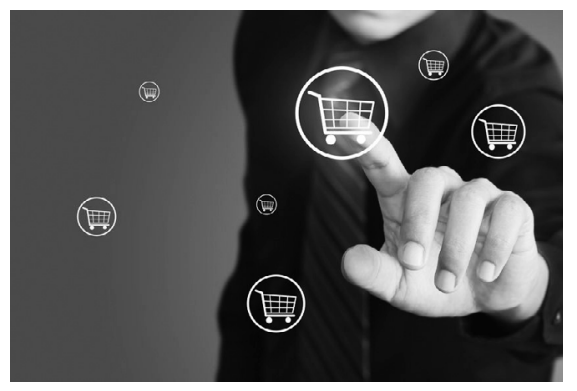

Der Einkauf verantwortet einen der großen Kostenblöcke in sozialen Unternehmen. Eine ganzheitliche Herangehensweise an das Thema umfasst das Bestellwesen, die Lagerung und Logistik, den strategischen Einkauf sowie die dazu gehörenden Verwaltungsprozesse.
Ziele eines effizienten Einkaufswesens

- Gesicherte Qualität

- Pünktliche Lieferungen

- Günstige Einkaufspreise

- Größtmögliche Effizienz

- Ausreichende Liquidität

- Angemessener Cashflow

- Geringe Kapitalbindung

Gangbare Wege zu den Zielen

- Effiziente Prozesse und Strukturen

- Geringe Lagerbestände

- Bestmögliche Einkaufskonditionen

- Standardisierte Produkte und Dienstleistungen

- E-Sourcing

Gabriele Heller

www.heller-consulting.net 
Die Anwendbarkeit des Systems bringt kleineren und mittleren Sozialträgern zusätzlich noch den Vorteil, dass die Größenordnung des Gesamteinkaufs ihre jeweilige Marktmacht in allen Bereichen wesentlich übersteigt und in der Regel erhebliche Kostenreduktionen möglich macht, die dank Preisbindungsfristen plan- und kalkulierbar sind und den Einkauf von der Notwendigkeit der täglichen Verhandlungsoptimierung mit den Lieferanten befreit.

\section{Fazit}

Kleiner Test: Wie lange brauchen Sie für die Antwort der Frage, wie viel Toilettenpapier Sie im Jahr zu welchem Preis verbrauchen? Benötigen Sie hierfür einen Tastendruck, bis in die Artikelebene hinein und mit Angabe der Verbrauchswerte einzelner Standorte?

Wenn auch in sozialen Unternehmen Beschaffung bisher ein Randthema war, das von einer »Drittel-Stelle « oder von der Einrichtungsleitung mitorganisiert wird, lohnt es sich, den Einkauf auf eine strukturierte Methode umzustellen.

In der Regel verläuft diese Umstellung intern kostenneutral, bringt aber neben Kosteneffekten in der Beschaffung auch andere wesentliche Vorteile. Angesprochen werden hier die Transparenz zur Erfüllung von Compliance-Anforderungen intern und extern, die Anschlussmöglichkeit an die weitere Digitalisierung der Buchführung und die Freisetzung von personellen Ressourcen, die für die inhaltliche Arbeit im Unternehmen dringend benötigt wird.

\section{Sozialplanung als Instrument für bedarfsgerechtes Ressourcenmanagement}

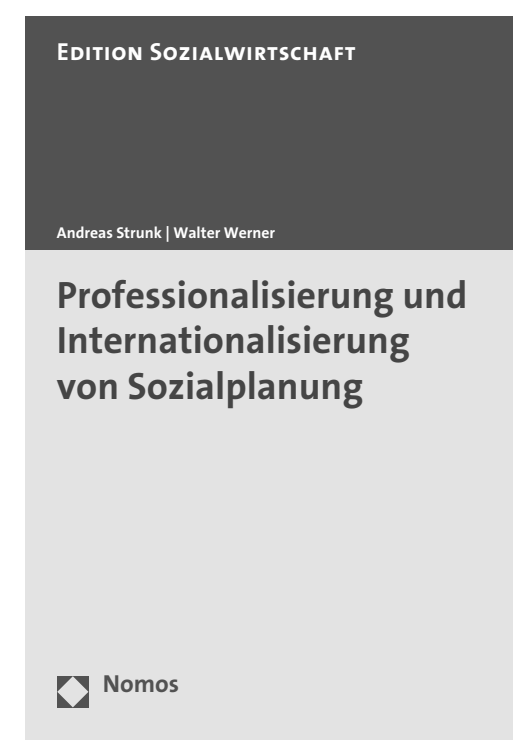

\section{Professionalisierung und Internationalisierung von Sozialplanung}

Von Prof. Dr. Andreas Strunk und Walter Werner

2019, 204 S., brosch., 39,-€

ISBN 978-3-8487-4142-7

(Edition Sozialwirtschaft, $B d$. 44)

nomos-shop.de/29818

Der neue Band zur Sozialplanung beantwortet die wichtigsten Fragen zu den relevanten von der Sozialplanung betroffenen Bereichen wie unter anderem Jugendhilfe, internationale Sozialplanung und Stadtentwicklung. Dabei liegt die Stärke des Bandes in der Verbindung von wissenschaftlich orientierten und von praktischen Erfahrungen geprägten Berichten, die jeweils die aktuellsten Ansätze aufgreifen.

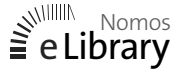

Bestellen Sie jetzt telefonisch unter (+49)7221/2104-37. Portofreie Buch-Bestellungen unter www.nomos-shop.de Nomos Alle Preise inkl. Mehrwertsteuer 